
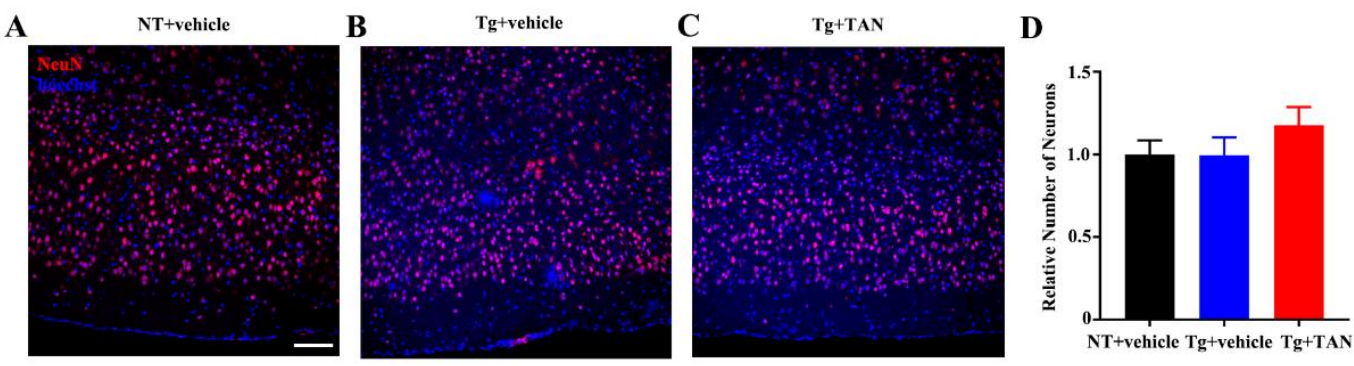

2 Figure S1. The number of neurons in cortex not changed after TAN administration. (A,

3 B and C) Representative immunofluorescence images of NeuN in NT+vehicle (A),

4 Tg+vehicle (B), or Tg+TAN (C) mice. Red: NeuN, blue: hoechst, scale bar $=100 \mu \mathrm{m}$.

5 (D) Quantification of relative number of neurons. Data are presented as mean \pm SEM,

$6 \mathrm{n}=3-4 /$ group.

7

8

9 
A

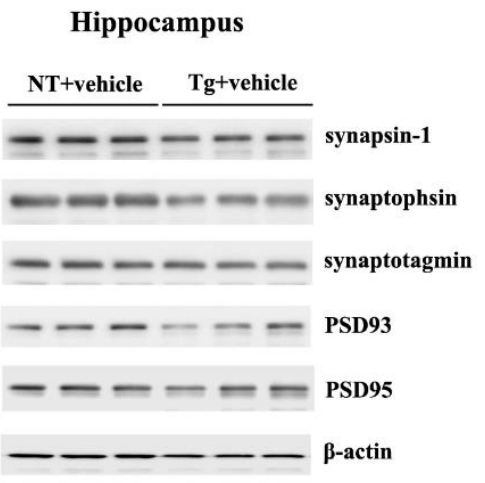

C

Cortex

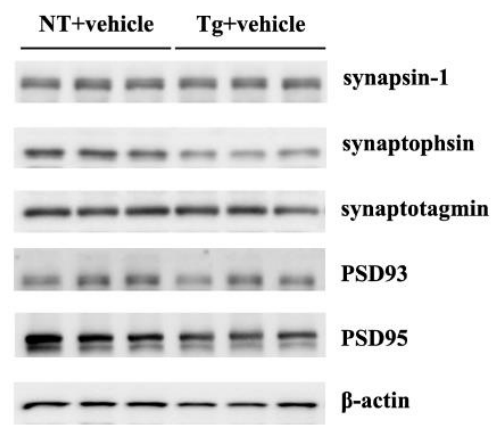

B

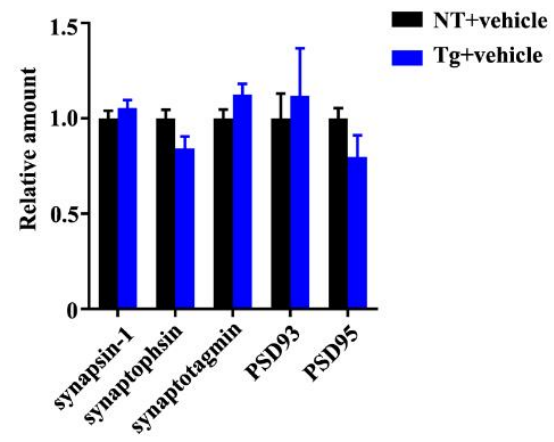

D

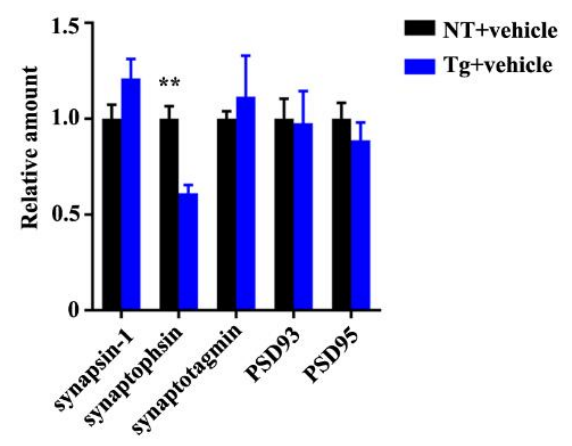

2 Figure S2. The levels of synaptic proteins increase in the hippocampus and cortex of

3 wide-type and APP/PS1 mice after vehicle administration. (A and B) Western blotting

4 and quantification of pre-synaptic proteins, such as Synapsin-1, Synaptophsin,

5 Synaptotagmin and post-synaptic proteins, such as PSD93, and PSD95 in the

6 hippocampus of NT+vehicle or Tg+vehicle mice. (C and D) Western blotting and

7 quantification of pre-synaptic proteins, such as Synapsin-1, Synaptophsin,

8 Synaptotagmin and post-synaptic proteins, such as PSD93, and PSD95 in the cortex of

$9 \mathrm{NT}+$ vehicle or Tg+vehicle mice. Data are presented as mean \pm SEM, $\mathrm{n}=3$ /group. $* * \mathrm{p}$

$10<0.01$ 

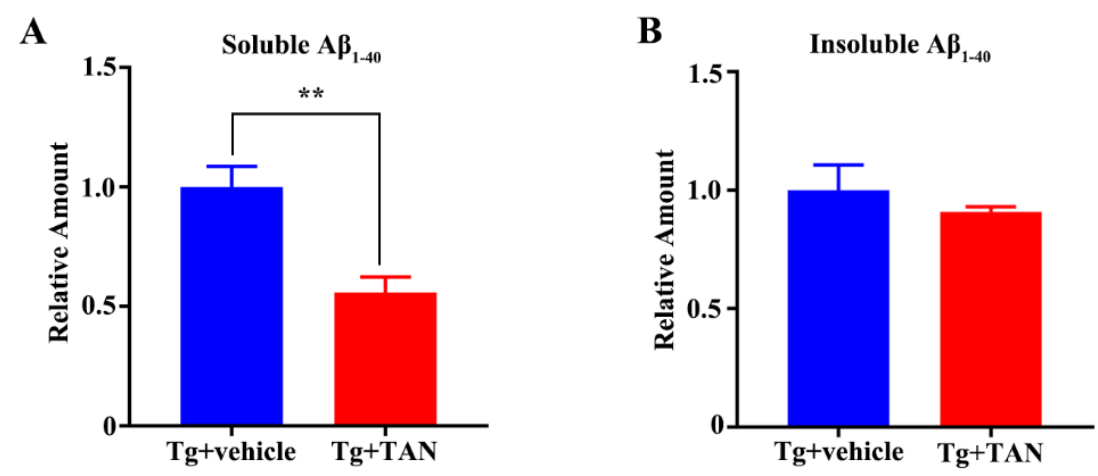

1

2 Figure S3. (A-B) The soluble and insoluble $A \beta_{1-40}$ levels were measured by ELISA. $n$ $3=4$ group. $* * \mathrm{p}<0.01$.

4

5

6

7

8

9

10

11

12

13

14 

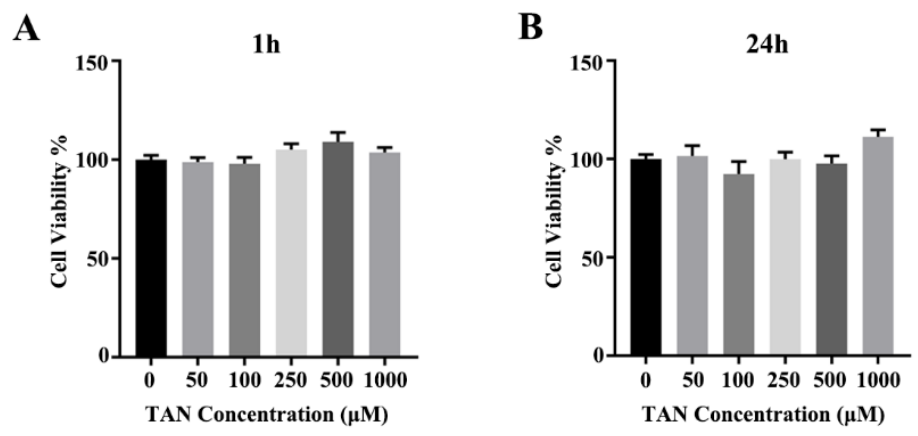

1

2

3

4

5

6

7

8

10

11

12

Figure S4. TAN exposure at concentrations of up to $1000 \mu \mathrm{M}$ for $1 \mathrm{~h}$ or $24 \mathrm{~h}$ exerted no effect on HEK 293T cell viability, $n=5 /$ group.

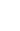

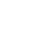

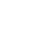

(1)

(1)

\section{9 9}


A

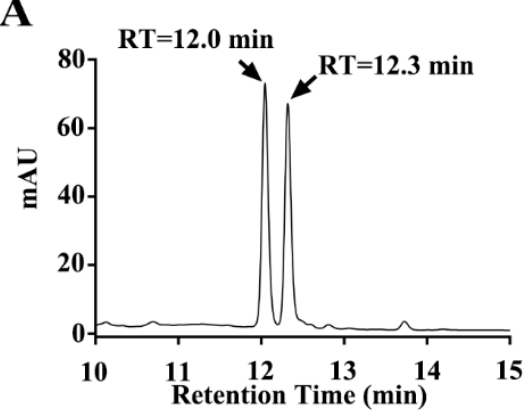

B

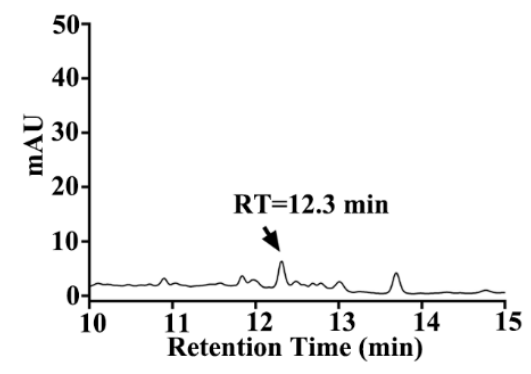

C

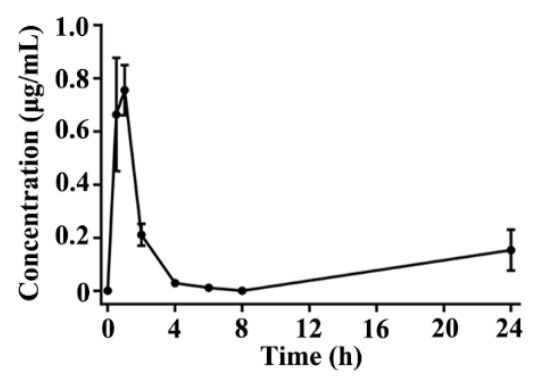

1

2 Figure S5. The presence of TAN was detected in the brain after oral administration. (A)

3 HPLC analysis of the TAN standards (retention time at $12.3 \mathrm{~min}$ ) and nobiletin

4 standards (retention time at $12 \mathrm{~min}$ ). (B) A brain sample after oral administration of

5 TAN, the retention time of TAN is $12.3 \mathrm{~min}$. (C) Concentration-time profiles of TAN

6 in brain after oral administration of $100 \mathrm{mg} / \mathrm{kg}$ TAN. Data are presented as mean \pm

$7 \quad$ SEM, $\mathrm{n}=3$ for each time point.

8 